\title{
A Novel Degradation Feature Extraction Technique Based on Improved Base-Scale Entropy
}

\author{
Zhenyi Chen \\ Logistics Engineering College, Shanghai Maritime University, Shanghai, 201306 China.

\section{Changzhuan Shao} \\ College of Arts and Sciences, Shanghai Maritime University, Shanghai, 201306 China. \\ Xiong Hu, Bing Wang and Daobing Zhang \\ Logistics Engineering College, Shanghai Maritime University, Shanghai, 201306 China.
}

\author{
Xiaomei Tao \\ Vocational College, Shanghai Jian Qiao University, Shanghai, 201306 China.
}

\begin{abstract}
(Received 23 May 2020; accepted 18 December 2020)
In order to track the performance degradation trend accurately, a novel degradation feature extraction technique is proposed based on improved base-scale entropy. A unified base scale is proposed and a new symbol standard is defined to overcome the disadvantages of the base-scale entropy method, so as to symbolize signal amplitude to characterize information amount under different degradation conditions quantitatively. A lifetime dataset of rolling bearing from the IMS Bearing Experiment Center is introduced. For instance, analysis and some entropybased techniques including fuzzy entropy, approximate entropy and sample entropy are imported for comparison. The results show that the improved basic-scale technique is able to characterize information amount of the signal amplitude distribution, so that the characterizing performance degradation degree of bearing shows a proportional relationship. When comparing the entropy-based techniques, the improved base-scale entropy technique has a faster calculation speed and better algorithm stability.
\end{abstract}

\section{INTRODUCTION}

Rolling bearings are key supporting components for a gearbox, motor and other equipment. Its overall health condition directly influences the operating status of the equipment. Slow performance degradation or sudden failure will occur under the long-term influence of the operating environment, ${ }^{1}$ leading to downtime or even casualties. The degradation trend will be tracked accurately if the vibration signals can be monitored online and analyzed effectively. Also a foundation of Condition-Based Maintenance ( CBM) is able to be utilized, thus the safety and reliability of the equipments will be improved. ${ }^{2}$

Feature extraction, known as the foundation of degradation condition assessment, is the critical step of the CBM technique. The main target for feature extraction is the mining health indicator which is able to reflect the performance degradation trend quantitatively and accurately. At present, linear analysis methods based on time domain, frequency domain and timefrequency domain analysis are widely used, ${ }^{3-5}$ some healthy indicators are proposed including spectral kurtosis, ${ }^{6,7}$ spectral L2/L1 norm, ${ }^{8}$ kurtosis ${ }^{9}$ and so on. Considering the nonlinear and non-stationary character of mechanical vibration signals, the complexity analysis method based on entropy and fractal dimension information is frequently employed in feature analysis of rotating machinery such as bearings and gears. In recent years, these methods included: modified multi-scale symbolic dynamic entropy, ${ }^{10}$ Local Mean Decomposition (LMD) sample entropy, ${ }^{11}$ multi scale permutation entropy, ${ }^{12,13}$ morphological fractal dimension ${ }^{14}$, etc.

As a typical complexity analysis method, base-scale entropy
(BSE) is a typical non-linear analysis technique with the advantages of a simple calculation and strong anti-interference ability. It is usually applied in an ECG signal analysis ${ }^{15,16}$ currently. There are still some preliminary applications in the fault diagnosis field. ${ }^{17,18}$ As to the degradation feature extraction field in this paper, there are relatively few studies. The reason seems to be that indicators based on the BSE technique have few relationships with the degradation trend, which means the BSE value has no apparent increasing or decreasing tendency when the degradation degree deepens, resulting in the difficulty in describing the degradation degree accurately.

According to its disadvantages in the degradation feature description, the BSE technique is improved and named improved base-scale entropy technique (IBSE) and proposed. A unified base scale (abbreviated as $B S_{0}$ ) is imported in IBSE for signal amplitude symbolization to characterize information amount of diverse degradation degree. The validity is analyzed using the bearing lifetime data of the Intelligence Maintenance System (IMS) as the data source.

The paper is organized as follows: Section 2 introduces some entropy-based techniques. Section 3 introduces improvement of the base-scale entropy technique. In Section 4, the technique is verified and the results are discussed. Finally, the conclusion of this paper is given in Section 5.

\section{BASIC THEORY}

In this section, some entropy-based techniques are elaborated for contrasting comparison in the following sections. 


\subsection{Approximate Entropy}

Approximate entropy can be used to measure the complexity and irregular nature of the system. The measurement formula is defined as $\operatorname{ApEn}(m, r, N)$, where $m$ is the embedding dimension and $r$ means the similarity tolerance, $N$ represents the number of time series points. Assuming that the original time series are $x(1), x(2), \ldots, x(N)$, the approximate entropy can be calculated in the following steps:

(1) Converting time series to an $m$-dimensional vector as follows:

$$
\begin{array}{r}
X(i)=[x(i), x(i+1), x(i+2), \ldots, x(i+m+1)], \\
1 \leq i \leq N-m+1 .
\end{array}
$$

(2) Defining the maximum value of the absolute difference between each vector as the distance $d$. For example, distance between vectors $\mathrm{X}(i)$ and $\mathrm{X}(j)$ is defined as $d[\mathrm{X}(i), \mathrm{X}(j)]$ as follows:

$$
\begin{gathered}
d[X(i), X(j)]=\max _{k=1, \ldots, m-1}|x(i+k)-x(j+k)| \\
i \geq 1, j \leq N-m+1, i \neq j .
\end{gathered}
$$

(3) When the similarity tolerance $r$ is given, $M^{m}(i)$ represents vector $\mathrm{x}(i)$ meeting $d[\mathrm{X}(i), \mathrm{X}(j)]<=r$, and $C_{r}^{m}(r)$ is defined as follows for $i=N-m+1$.

$$
C_{r}^{m}(i)=\frac{M^{m}(i)}{N-m+1} .
$$

(4) Calculating natural logarithm of $C_{r}^{m}(i)$ and find the average value.

$$
\phi^{m}(r)=\frac{1}{N-m+1} \sum_{i=1}^{N-m+1} \ln C_{r}^{m}(i) .
$$

(5) Changing the above dimension from $m$ to $m+1$, and repeating steps (1) to (4) to get $C_{r}^{m+1}(i)$ and $\phi_{i}^{m+1}(i)$.

(6) Calculating the Approximate entropy as follows.

$$
\operatorname{ApEn}(m, r, N)=\phi^{m}(r)-\phi^{m+1}(r) .
$$

\subsection{Sample Entropy}

The sample entropy is an improved algorithm of approximate entropy, which is able to determine the irregular degree of time series in a certain dimension. The specific algorithm of the sample entropy is as follows:

(1) Time series $x(1), x(2), \ldots, x(N)$ are formed into mdimensional vectors.

$$
\begin{array}{r}
X_{m}(i)=[x(i), x(i+1), \ldots, x(i+m-1)] \\
1 \leq i \leq N-m+1 .
\end{array}
$$

(2) Defining the largest difference among the vectors as distance $d$.

$$
\begin{gathered}
d\left[X_{m}(i), X_{m}(j)\right]=\max |x(i+k)-x(j+k)| \\
1 \leq k \leq m-1 ; 1 \leq i, j \leq N-m+1, i \neq j .
\end{gathered}
$$

(3) Given a threshold $r$, counting the number of $d\left[\mathrm{x}_{\mathrm{m}}(i)\right.$, $\left.\mathrm{x}_{\mathrm{m}}(j)\right]<\mathrm{r}$ (called the number of template matches), making a ratio of the number and $N-m$-1as follows.

$$
\begin{gathered}
B_{i}^{m}(r)=\frac{1}{N-m-1} \operatorname{num}\left\{d\left[X_{m}(i), X_{m}(j)\right]<r\right\} \\
1 \leq j \leq N-m, j \neq i .
\end{gathered}
$$

Calculating average value as follows.

$$
B^{m}(r)=\frac{1}{N-m} \sum_{i=1}^{N-m} B_{i}^{m}(r) .
$$

As shown above: $A_{i}^{m}(r)$ and $A^{\mathrm{m}}(r)$ can be also calculated following the formula.

$$
\begin{gathered}
A_{i}^{m}(r)=\frac{1}{N-m} \operatorname{num}\left\{d\left[X_{m+1}(i), X_{m+1}(j)\right]<r\right\} \\
1 \leq j \leq N-m, j \neq i \\
A^{m}(r)=\frac{1}{N-m} \sum_{i=1}^{N-m} A_{i}^{m}(r) .
\end{gathered}
$$

(4) The sample entropy of this sequence is calculated as follows:

$$
\operatorname{SampEn}(m, r, N)=\lim _{N \rightarrow \infty}\left\{-\ln \left[A^{m}(r) / B^{m}(r)\right]\right\} .
$$

The precise value can be estimated as follows when $N$ takes a finite value.

$$
\operatorname{SampEn}(m, r, N)=-\ln \left[A^{m}(r) / B^{m}(r)\right] .
$$

\subsection{Fuzzy Entropy}

On the basis of the sample entropy algorithm, the fuzzy entropy is proposed and has the character of stable results and strong noise resistance. The algorithm is as follows.

(1) Reconstructing the phase space of the sequence $\{\mu(i): 1 \leq i \leq N\}$ into an $m$-dimensional vector where $i=1,2, \ldots, N-m$

$$
X_{i}^{m}=\left\{\mu(i), \mu(i+1), \ldots, \mu(i+m-1)-\mu_{0}(i)\right\} .
$$

$\mu(i)$ is defined as mean value as the following formula.

$$
\mu_{0}(i)=\frac{1}{m} \sum_{j=0}^{m-1} \mu(i+j)
$$

(2) Defining the largest difference between two vectors as the distance $d_{i j}^{m}$.

(3) Defining the similarity of the vectors $X_{i}^{m}$ and $X_{j}^{m}$ based on the fuzzy function.

$$
D_{\mathrm{ij}}^{m}=\mu\left(d_{\mathrm{ij}}^{m}, n, r\right)=\exp \left(-\frac{d_{\mathrm{ij}}^{m^{n}}}{r}\right) .
$$

Among them, the fuzzy function is an exponential function, and $n$ represents the boundary gradient of the function, $r$ means the boundary width of the function.

(4) Defining the function as follows.

$$
\phi^{m}(n, r)=\frac{1}{N-m} \sum_{i=1}^{N-m}\left(\frac{1}{N-m-1} \sum_{j=1, j \neq i}^{N-m} D_{\mathrm{ij}}^{m}\right) .
$$

(5) Repeating step 1 to step 4 above and generating an $m+1$ dimensional vector, the function is defined as follows.

$$
\phi^{m+1}(n, r)=\frac{1}{N-m} \sum_{i=1}^{N-m}\left(\frac{1}{N-m-1} \sum_{j=1, j \neq i}^{N-m} D_{\mathrm{ij}}^{m+1}\right) .
$$


(6) The fuzzy entropy can be calculated as follows.

$\operatorname{FuzzyEn}(m, n, r, N)=\lim _{N-\infty}\left(\ln \phi^{m}(n, r)-\ln \phi^{m+1}(n, r)\right)$.

The precise value can be estimated as follows when $N$ takes a finite value.

$$
\operatorname{FuzzyEn}(m, n, r, N)=\ln \phi^{m}(n, r)-\ln \phi^{m+1}(n, r) .
$$

\subsection{Root Mean Square}

The root mean square ( RMS) is also called the effective value. Assuming the time series is $x(1), x(2), \ldots, x(N)$, then the root mean square of the series is calculated as follows:

$X_{\mathrm{rms}}=\sqrt{\frac{\sum_{i=1}^{N} X^{2}(i)}{N}}=\sqrt{\frac{X^{2}(1)+X^{2}(2)+\ldots+X^{2}(N)}{N}}$.

\section{IMPROVEMENT OF ENTROPY}

\subsection{Base-Scale Entropy}

The named base-scale entropy technique is able to describe the complexity of time series ${ }^{19}$ and the principle is summarized as follows.

Supposing $u$ is a one-dimensional time series with length $N$, it is transformed into an $m$-dimensional vector $X$ following phase space reconstruction firstly.

$$
X(i)=[u(i), u(i+L), \ldots, u(i+(m-1) L)] .
$$

In the formula above, $m$ is the vector dimension and $L$ represents delay factor meeting $i+(m-1) L \leq N$. The value of $L$ is usually set as 1 and then $X$ is a $m^{*}(N-m+1)$ structuring matrix.

Secondly, each $m$-dimensional vector in matrix $X$ is sequentially symbolized to form an $m$-dimensional symbol sequence $S$.

$$
S_{i}\left(X_{i}\right)=\{s(i), s(i+L), \ldots, s(i+(m-1) L)\} .
$$

In the formula above, $s$ means the four symbols meeting $s \in$ $A: A=0,1,2,3$ and the symbolic rules are as follows.

$$
S_{i}\left(X_{i}\right)=\left\{\begin{array}{l}
0: \bar{u}<u_{i+k} \leq \bar{u}+a \times B S \\
1: u_{i+k}>\bar{u}+a \times B S \\
2: \bar{u}-a \times B S<u_{i+k}<\bar{u} \\
3: u_{i+k} \leq \bar{u}-a \times B S
\end{array} .\right.
$$

In the formula, parameter $a$ is defined as a base scale parameter which is important to split the difference of the main tendency and detail. Parameter $u$ is the average value of the $i^{\text {th }} m$-dimensional symbol sequence. $B S$ means the base scale of the $i^{t h} m$-dimensional symbol sequence which is defined as mean root value of the adjacent points. The calculation method of $B S$ is as follows.

$$
B S(i)=\sqrt{\frac{\sum_{j=1}^{m-1}[u(i+j)-u(i+j-1)]^{2}}{m-1}} .
$$

Thirdly, the distribution probability $P$ of different symbol sequence is counted as follows in which $\pi$ is some arrangement mode of the symbol sequence with a maximum value of $4^{m}$.
The parameter $t$ is the symbol vector's serial number meeting $1 \leq t \leq N-m+1$, and \# represents the count number of $\pi$.

$$
p(\pi)=\frac{\#\left(u_{t}, u_{t+1}, \ldots, u_{t+m-1}\right)}{N-m+1}, 1 \leq t \leq N-m+1
$$

Finally, base-scale entropy of $u$ is calculated as follows.

$$
\operatorname{bse}(m)=\frac{-\sum p(\pi) \log _{2} p(\pi)}{\log { }_{2}\left(4^{m}\right)} .
$$

In general, parameter $m$ is set as integer between 3 and 7, and the value of $b s e$ is proportional to the complexity of time series.

\subsection{Improved Base-Scale Entropy}

In the calculation process of BSE technique, $\mathrm{a} \times B S$ is introduced as the symbol standard in formula (24), that means we should calculate $B S$ for each $m$-dimensional vector and choose a proper parameter $a$ for symbol sequence construction, which will bring a huge calculation amount and instability factors.

The IBSE is introduced by changing the symbol standard above. The calculation process of IBSE is summarized as follows.

Transforming time series into an $m$-dimensional vector $X$ following the phase space reconstruction firstly.

$$
X(i)=[u(i), u(i+L), \ldots, u(i+(m-1) L)] .
$$

Secondly, each $m$-dimensional vector is sequentially symbolized to form an $m$-dimensional symbol sequence $S$ as the following standard in which parameter $s$ meets $s \in A: A=$ $0,1,2,3,4,5$.

$$
\begin{aligned}
& S_{i}\left(X_{i}\right)=\{s(i), s(i+L), \ldots, s(i+(m-1) L)\} . \\
& S_{i}\left(X_{i}\right)=\left\{\begin{array}{l}
1,|u(i)| \leq B S_{0} \\
2, B S_{0}<|u(i)| \leq 2 \times B S_{0} \\
3,2 \times B S_{0}<|u(i)| \leq 3 \times B S_{0} \\
4,3 \times B S_{0}<|u(i)| \leq 4 \times B S_{0} \\
5,4 \times B S_{0}<|u(i)|
\end{array} .\right.
\end{aligned}
$$

$B S_{0}$ is the base scale for IBSE method in the formula above. It is a fixed parameter during the whole computing process compared with the BSE method in which $\mathrm{a} \times B S$ is $a$ variable for each $m$-dimensional vector. The proposed IBSE technique has advantages of a small calculation amount and strong antinoise ability.

On the basis above, the third and final step will be applied and the improved base-scale entropy of $u$ will be calculated as $i b s e$, the value of which describes the degradation degree.

\section{INSTANCE ANALYSIS}

In this section, we chose a typical lifetime vibration signal of rolling bearing, extracting the degradation feature, calculating the health indicators based on BSE and IBSE techniques, and analyzing the effect of the health indicators in describing degradation degree. 


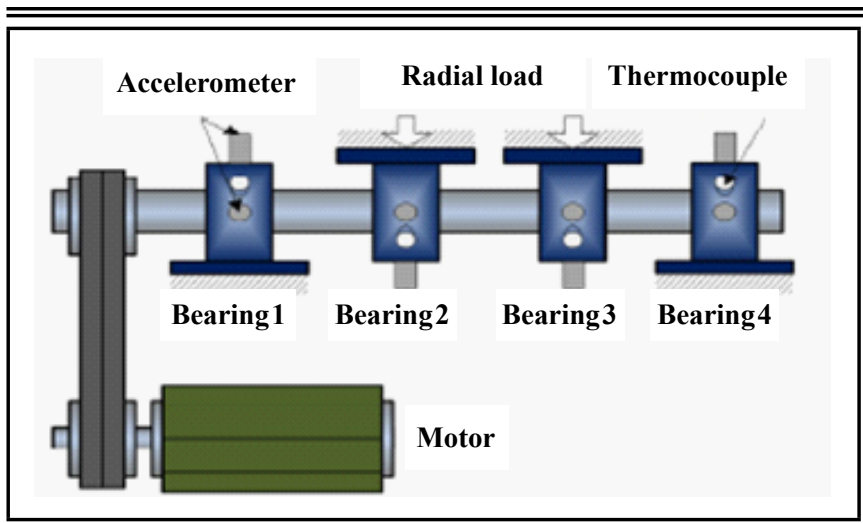

Figure 1. Schematic of accelerated test bench.

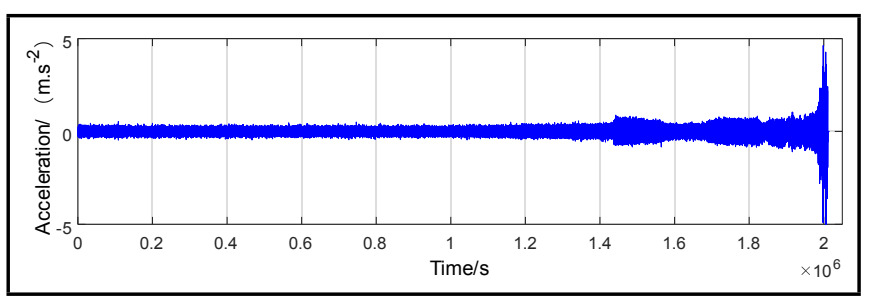

Figure 2. Time domain waveform of lifetime dataset.

\subsection{Bearing Lifetime Test}

A rolling bearing lifetime dataset from the University of Cincinnati's IMS is introduced for instant analysis. The schematic diagram of accelerated life test bench is shown in Fig. $1 .^{20}$

In the test bench, four sets of double-row cylindrical rolling bearings are installed on the shaft, and the type is Rexnord ZA-2115 with 16 rollers in each row. The rolling group pitch diameter is $75.501 \mathrm{~mm}$ and the rolling element diameter is $8.4074 \mathrm{~mm}$ with pressure angle is $15.17^{\circ}$. The speed of the shaft ran at $2000 \mathrm{rpm}$ and a radial load of $2721.554 \mathrm{~kg}$ is loaded on the shaft by a spring device. Each bearing block is equipped with two high-sensitivity PCB acceleration sensors to collect vibration signals. The sampling frequency is $20 \mathrm{kHz}$, the sampling time of each group is $1 s$ and sampling interval ran for ten minutes, which means we will make a signal sampling every ten minutes.

The test bench ran from 11:16:18 on February 12 to 06:22:39 on February 19, and it was found that 1\# bearing was faulty with an outer ring failure when the test stopped automatically. A group of vibration signals was collected including 982 groups of sampling signals. The remaining 2\#-4\# bearings are intact. The lifetime dataset is introduced for instant analysis and the time domain waveform is shown in Fig. 2. It was clear that the amplitude of the signal will increase with the sampling time and the phased pattern was apparent. It was necessary to extract a health indicator to describe the whole degradation process quantitatively.

\subsection{Vibration Signal Symbolization}

In this section, part of the time-domain waveform was taken as an example for symbolic analysis as seen in Eq. (30). The parameter of $B S_{0}$ is assumed as 0.1 in IBSE technique. The division of the original signal's amplitude following the standard grid is shown in Fig. 3(a), and the results of the symbolizations are shown in Fig. 3(b).

It is clear that the signal amplitude is divided by the standard grid with array $\left[-4 B S_{0},-3 B S_{0},-2 B S_{0}\right.$,$\left.B S_{0}, B S_{0}, 2 B S_{0}, 3 B S_{0}, 4 B S_{0}\right]$, and the symbolization results

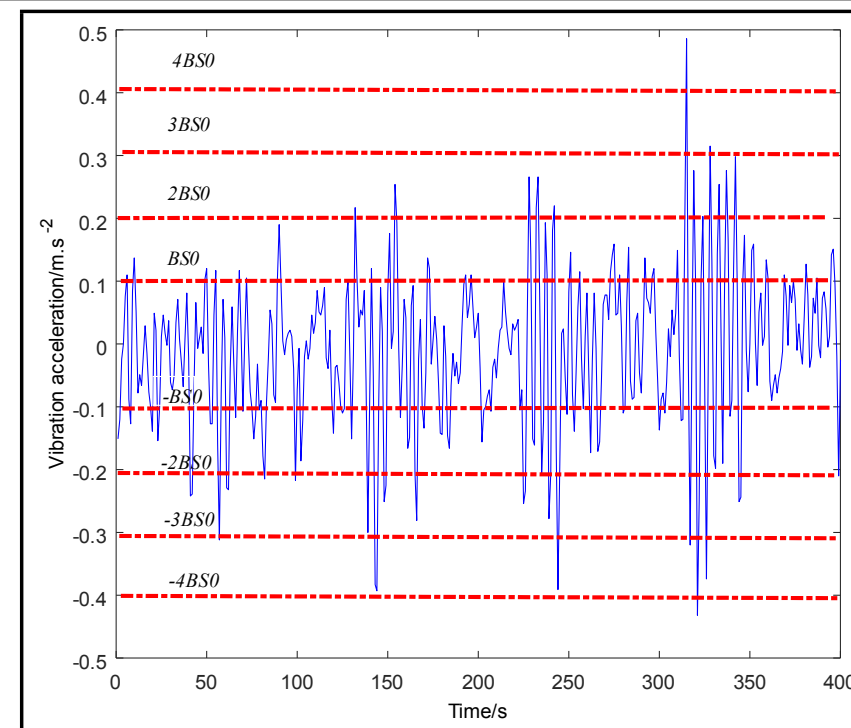

(a) Division of the signal amplitude

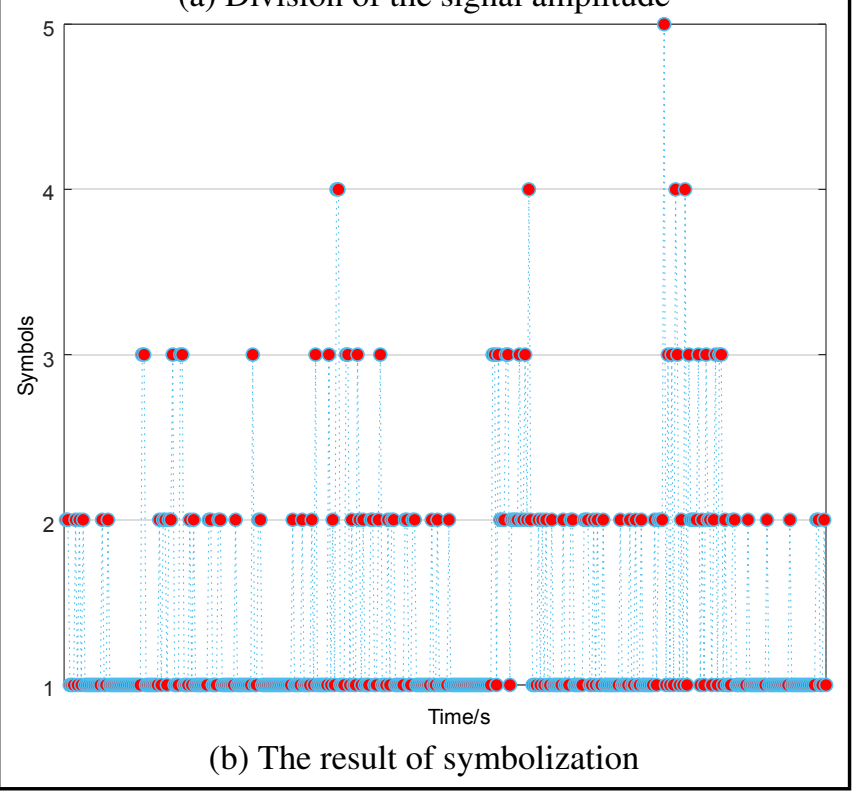

Figure 3. Vibration signal symbolization.

ran a coarse distribution information of an original sequence. According to the symbolization definition in Eq. (30), the amplitude within the interval $\left[-B S_{0}, B S_{0}\right]$ are uniformly symbolized to 1 so that noise interference in the original signal was able to be filtered out.

\subsection{Parameters Influence Analysis}

The two key parameters in the IBSE technique include $B S_{0}$ and $m$. This section will discuss the influence on IBSE value taking the $750^{\text {th }}$ group in lifetime dataset as an example, the waveform is shown in Fig. 4.

Considering that the value of $B S_{0}$ was able to affect the capability of the fluctuating filtering components, in this paper, root mean square (defined as $S_{0}$ ) of the signal on initial normal condition was adopted as the unit standard of the base scale. The relationship between the calculated $i b s e_{750}$ and $B S_{0}$ is shown in Fig. 5.

It is apparent that the $B S_{0}$ is inversely proportional to $i b s e_{750}$. The larger the value of $B S_{0}$, the lower the indicator's value. The main reason was that $B S_{0}$ directly determines the symbolization standard. The higher the base scale value, the fewer the number of symbolized modes and smaller was the distribution 


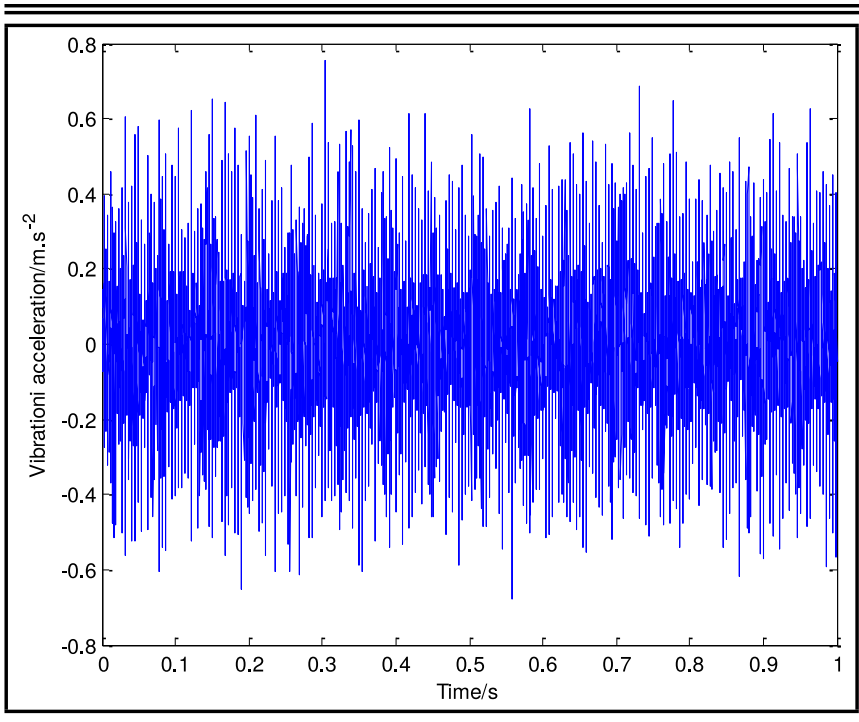

Figure 4. Waveform of the $750^{\text {th }}$ group.

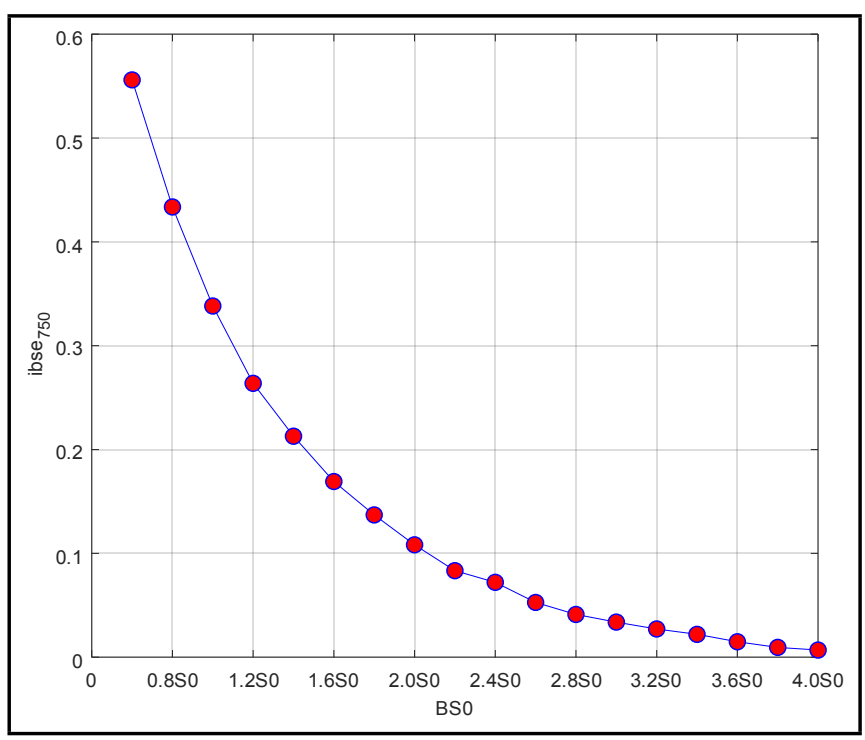

Figure 5. The correlation relation between $B S_{0}$ and $i b s e_{750}$.

entropy. In order to maintain high distinction for the indicator's value during the entire degradation process, $B S_{0}$ was set as 1.5 times the root mean square of the first group sampling signal in this paper, $\left(1.5 * S_{0}\right)$.

The effect of parameter $m$ on IBSE is shown in Fig. 6. The larger the value of $m$, the higher the obtained IBSE value. The value of IBSE tends to be stable when $m>16$.

However, the distinction of IBSE value will decrease during some sensitive periods in the whole degradation process and the calculation amount will increase rapidly. The comparison of IBSE value when $m=4$ and $m=16$ is shown in Fig. 7. As a result, in order to maintain a high distinction for IBSE value during the entire degradation process considering the calculation amount and speed, parameter $m$ is set as $m=4$ in this paper.

The effect on IBSE of the length of signal is shown in Fig. 8. It was apparent that the signal length will not influence the IBSE value, and it was not a key parameter for the IBSE technique.

\subsection{Degradation Feature Extraction Based on IBSE Technique}

Extracting degradation features using the IBSE technique on the lifetime dataset, the obtained IBSE degradation features are

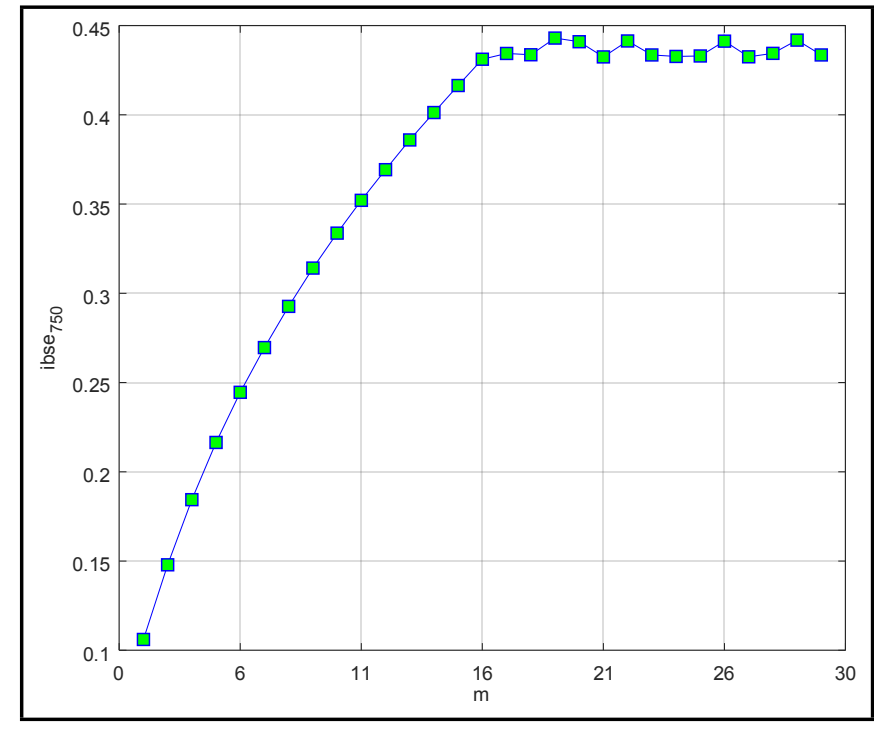

Figure 6. Correlation relation between $\mathrm{m}$ and IBSE.

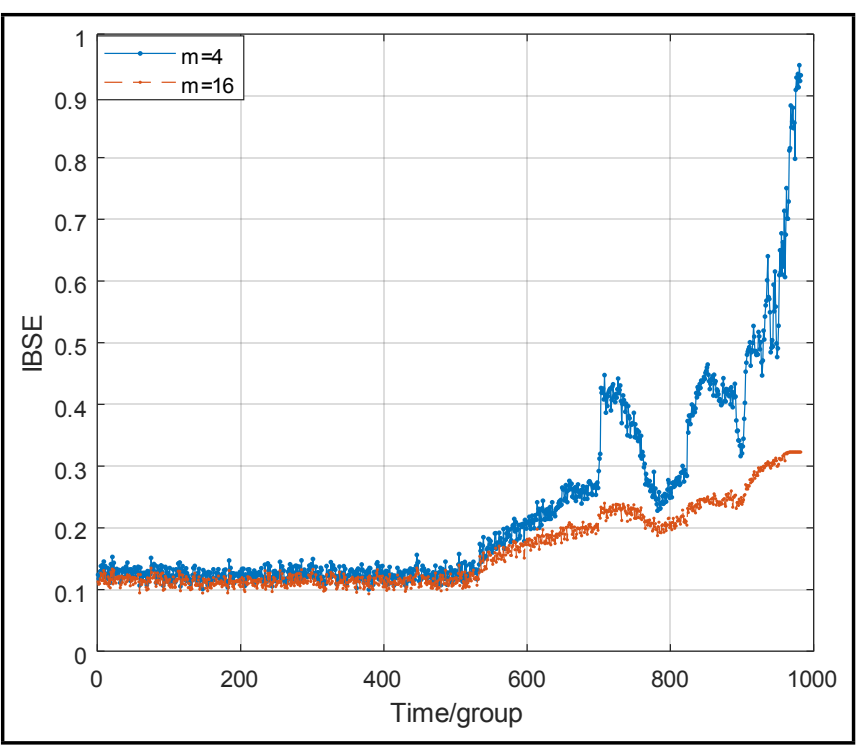

Figure 7. The comparison of IBSE value when $\mathrm{m}=4$ and $\mathrm{m}=16$.

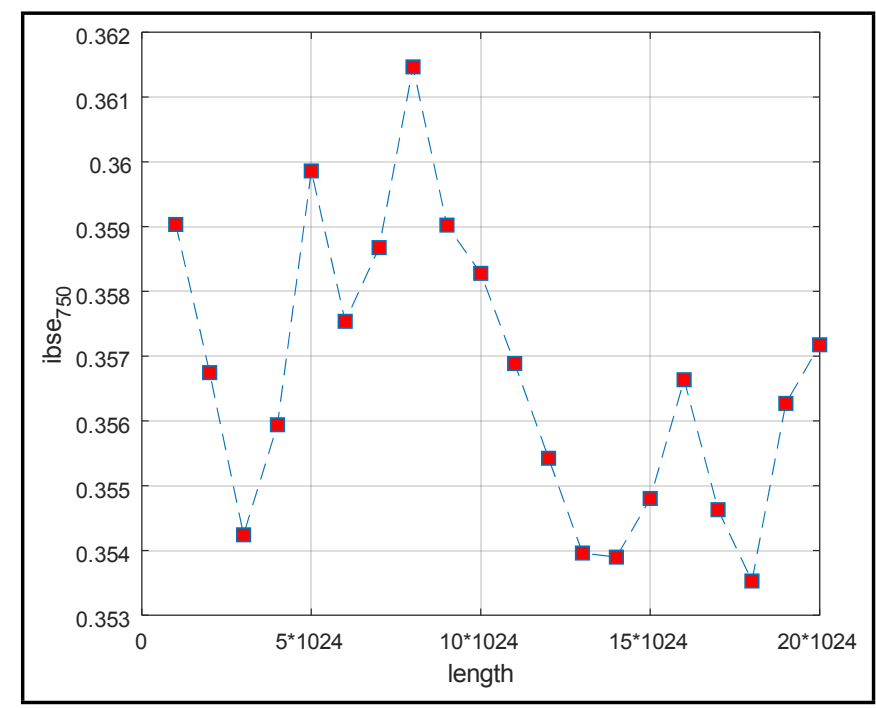

Figure 8. Correlation relation between the signal length and $i b s e_{750}$. 


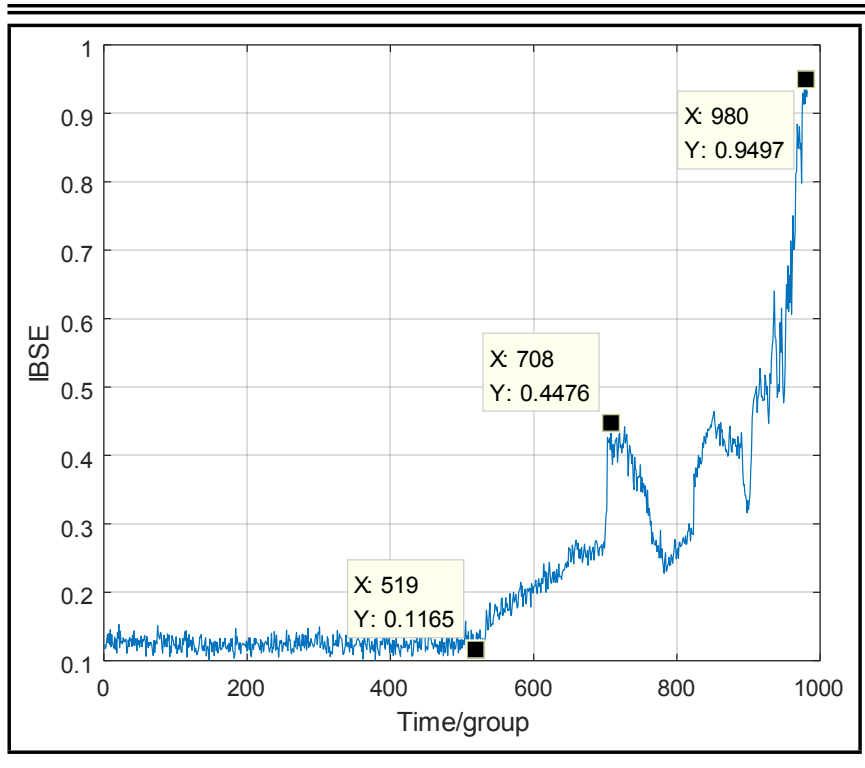

Figure 9. IBSE curve during the degradation process.

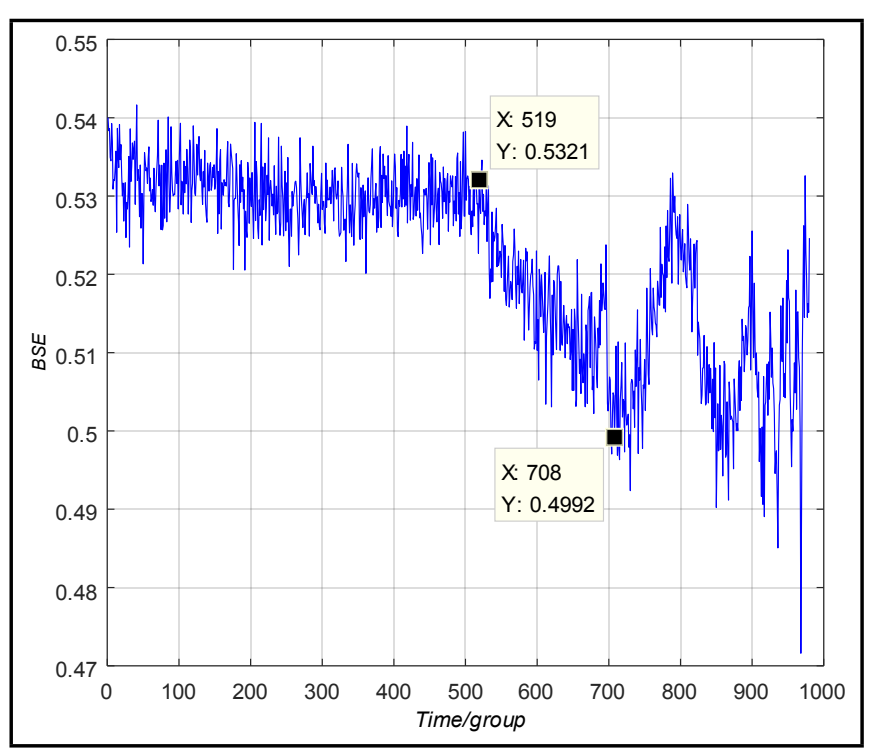

Figure 10. BSE curve during the degradation process.

shown in Fig. 9. It was clear that the IBSE feature increased as the degree of performance degradation deepened, while the whole trend of BSE shown in Fig. 10 was opposite to that of IBSE. Additionally, the IBSE curve shows apparent stages and low volatility as seen below.

In summary, it is evident from the comparison that this technique has weak relation with degradation trend, and after the improvement on the base scale and the symbolization standard, the IBSE technique was able to clearly reflect the performance degradation trend effectively.

The main reason for the opposite trend of IBSE and BSE degradation features was as follows: in the calculation process of the BSE technique, $a \times B S$ was important as the base scale for each group for which the amplitude distribution character was measured, so that the value of $B S$ changed accordingly. During the normal condition stage, the amplitude distribution was uniform and the obtained BSE value was the highest. As the degradation degree deepened, the impact and energy gradually increased and the amplitude distribution gradually became uneven. The BSE value gradually decreased accordingly. Correspondingly, the unified $B S_{0}$ was used as the base scale in the IBSE technique and the amplitude was symbolized based on
Table 1. Quantitative results of different techniques.

\begin{tabular}{|l|l|l|l||}
\hline Index & techniques & Time(s) & Variance \\
\hline 1 & IBSE & 0.049992 & $1.4331 \mathrm{e}-05$ \\
2 & BSE & 0.430447 & $4.3732 \mathrm{e}-05$ \\
3 & RMS & 0.012839 & $6.0416 \mathrm{e}-06$ \\
4 & fuzzy entropy & 0.933219 & $1.6280 \mathrm{e}-04$ \\
5 & approximate entropy & 0.674079 & $3.2514 \mathrm{e}-04$ \\
6 & sample entropy & 0.355605 & $8.2597 \mathrm{e}-04$ \\
\hline
\end{tabular}

$B S_{0}$, which quantitatively measured the information amount of all of the symbolized pattern distributions. It was found that the deeper the performance degradation, the more balanced the symbolization mode distribution. As a result, the value of IBSE was higher.

\subsection{Comparison}

In this section, the commonly used entropy-based techniques including approximate entropy, fuzzy entropy, sample entropy and root mean square were introduced for comparison with the IBSE technique proposed. Extracting degradation features on the whole lifetime dataset and the degradation features are shown in Fig. 11.

It is apparent that the value of entropy-based techniques is inversely proportional to degradation degree, which means that the deeper the degradation degree, the lower the value. The proposed IBSE and the Root mean square degradation feature reflect the amplitude distribution and energy accumulation respectively, so that the value is proportional to the degradation degree. The deeper the degradation degree is, the higher the value will be. The physical explanation on the inverse trends reveals that the entropy-based indicators are reflecting the entropy of signal distribution under a different definition quantitatively, and the IBSE indicator measures the information amount and energy accumulation so as to the RMS indicator.

At the time of the $500^{\text {th }}$ group, the rolling bearing showed a slight degradation tendency. At this time, sensitive changes occurred on the curves of the approximate entropy, sample entropy, fuzzy entropy, and the IBSE degradation feature, while the RMS degradation feature failed to reflect the tendency sensitivity because this indicator can only describe the statistical character.

Quantitative comparisons of different techniques are shown in Tab. 1. Average calculation time for each group and the variance of curve's stable interval $[0,500]$ are imported as the evaluation standards.

It is clear that the proposed IBSE technique has a faster operation speed than the BSE technique and other entropy-based techniques. The reason mainly is due to the unified symbolic standard $B S_{0}$ in the calculation process. Considering the stability, the IBSE technique is also superior to the entropy-based technique. Although the RMS indicator has the best calculation speed and variance, it is not sensitive to the slight degradation trend of the bearing performance.

As to the influence of parameters $B S_{0}$ and $\mathrm{m}$, the IBSE curves at different $B S_{0}$ are shown in Fig. 12 and the relationship between the IBSE curves and parameter $m$ is shown in Fig. 13. It is evident that the parameters will not change the whole evolution trend, which shows a certain stability of the proposed technique.

\section{CONCULUSION}

Upon examining the shortcomings of the base-scale entropy method including the lack of stability and slow calculation speed, an improved base-scale entropy technique was proposed 


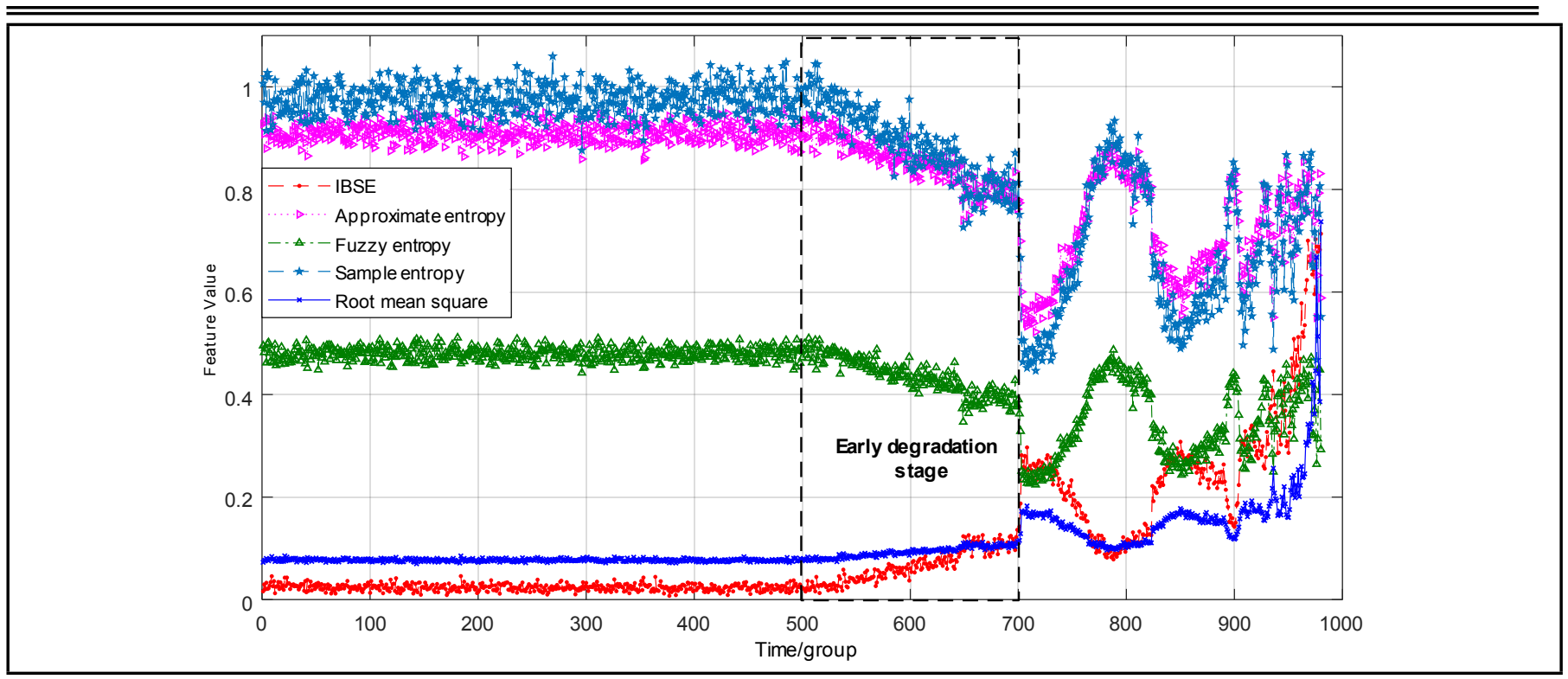

Figure 11. Comparison of different degradation features.

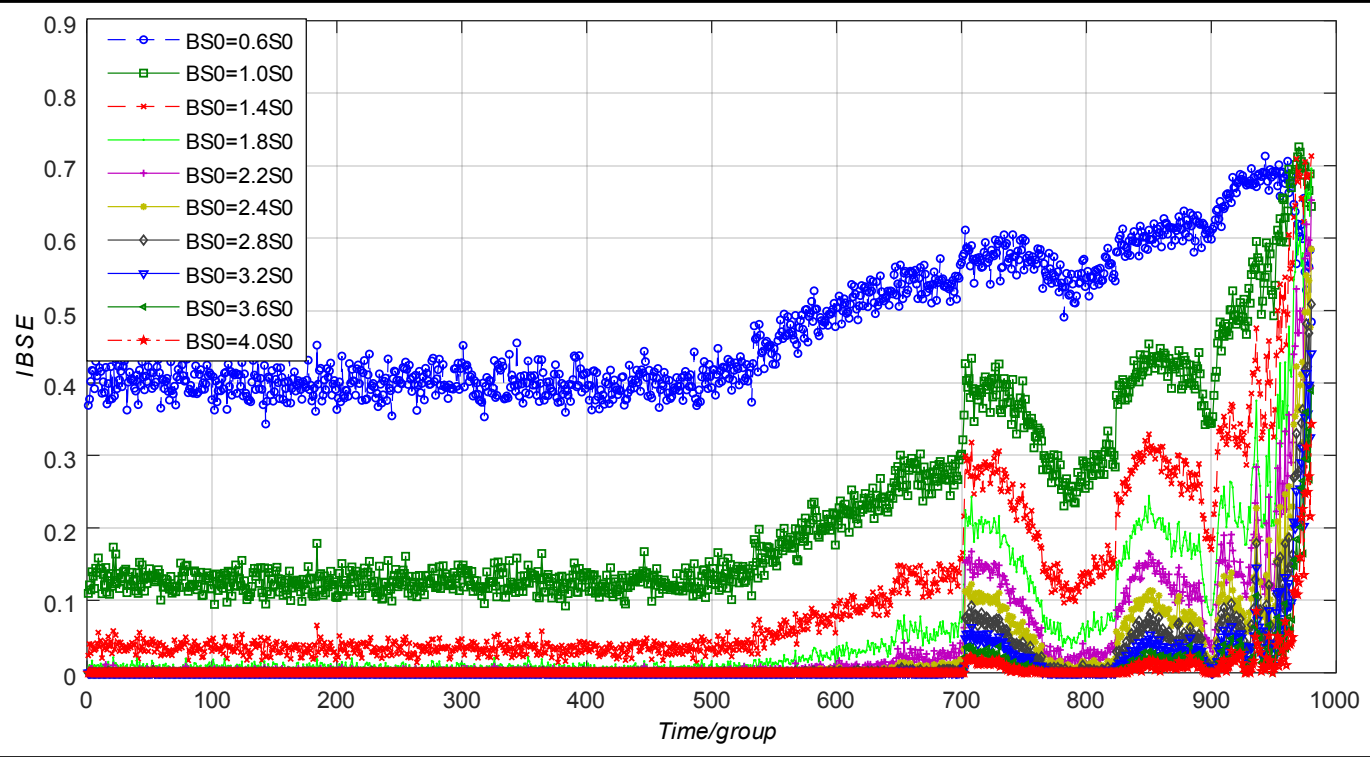

Figure 12. IBSE curve under different base scale.

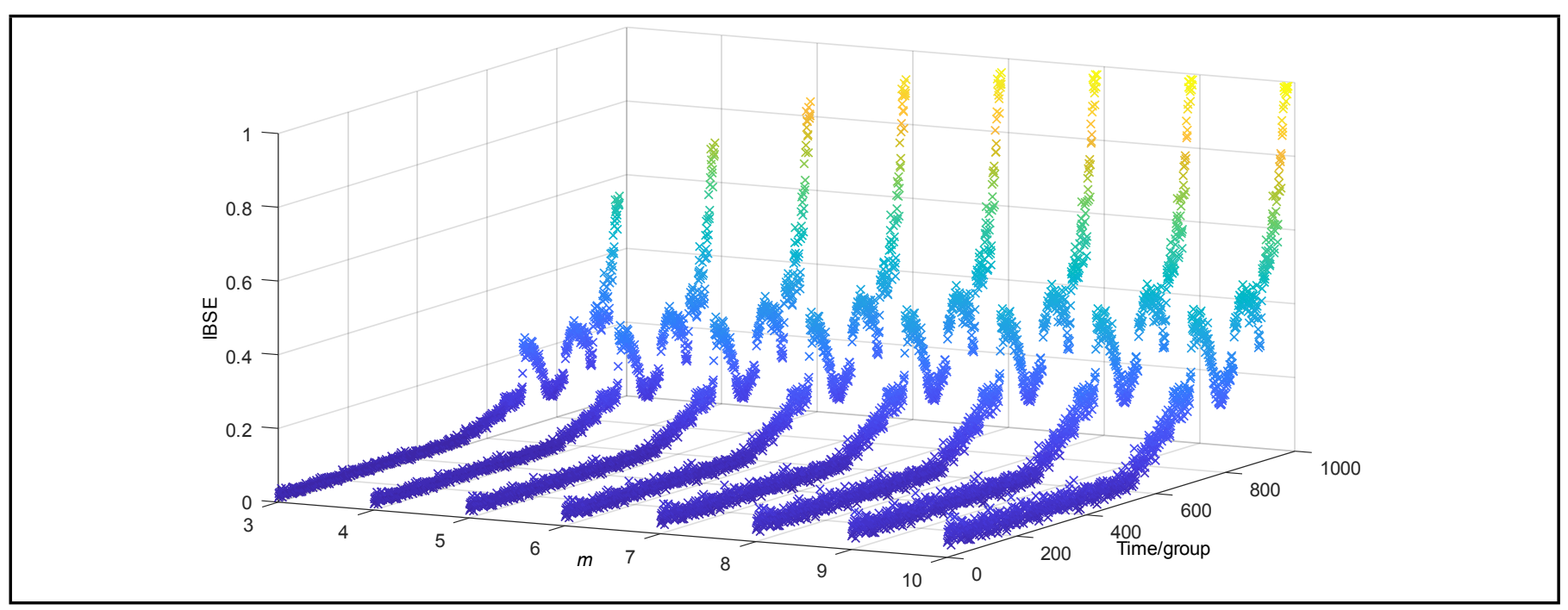

Figure 13. IBSE curve under different parameter $\mathrm{m}$. 
and applied in the degradation feature extraction for rolling bearings. The IMS lifetime dataset was introduced for verification. Some conclusions are as follows.

(1) Compared with the BSE algorithm, the improved basescale entropy was able to effectively describe the complexity of signal symbol sequence. The stronger the signal stability, the smaller the number of symbol patterns and the lower the IBSE value were observed. Conversely, the observation was made that the larger the number of symbol patterns, the larger the value.

(2) By introducing a unified basic scale $B S_{0}$, the algorithm's sensitivity to noise is able to be reduced and the calculation speed is accelerated. This can effectively describe the changing trend of signal symbol patterns.

(3) The value of the IBSE feature will gradually increase as health condition of the rolling bearing gradually degrades. This means the proposed IBSE technique was able to track the performance degradation process to a certain extent, which in turn lays a foundation for an online degradation feature extraction.

\section{ACKNOWLEDGEMENT}

The authors declare no conflict of interest in preparing this article.

\section{REFERENCES}

1 Nazmus, S. and Thorsten, W. Challenges and Opportunities of Condition-based Predictive Maintenance: A Review, ScienceDirect, 78, 267-272, (2018). https://dx.doi.org/10.1016/j.procir.2018.08.318

2 Li, H., Sun J., and Ma, H., et al. A novel method based upon modified composite spectrum and relative entropy for degradation feature extraction of hydraulic pump, Mechanical Systems \& Signal Processing, 114, 399-412, (2019). https://dx.doi.org/10.1016/j.ymssp.2018.04.040

3 Wang, B., Hu, X., and Li, H R. Rolling Bearing Performance Degradation Condition Recognition Based on Mathematical Morphological Fractal Dimension and Fuzzy C-Means, Measurement, 109, 109-115, (2017). https://dx.doi.org/10.1016/j.measurement.2017.05.033

4 Araneo, R., Attolini, G., and Celozzi, S., et al. TimeDomain Shielding Performance of Enclosures: A Comparison of Different Global Approaches, IEEE Transactions on Electromagnetic Compatibility, 58(2), 434-441, (2016). https://dx.doi.org/10.1109/TEMC.2015.2497742

5 Randall, R B. and Antoni, J. Rolling element bearing diagnostics-A tutorial, Mechanical Systems \& Signal Processing, 25(2), 485-520, (2011). https://dx.doi.org/10.1016/j.ymssp.2010.07.017

6 Zhao, L Y., Wang, L., and Yan, R Q. Rolling Bearing Fault Diagnosis Based on Wavelet Packet Decomposition and Multi-Scale Permutation Entropy, Entropy, 17, 64476461, (2015). https://dx.doi.org/10.3390/e17096447

7 Tian, J., Morillo, C., and Azarian, M H., et al. Motor Bearing Fault Detection Using Spectral KurtosisBased Feature Extraction Coupled With K-Nearest Neighbor Distance Analysis, IEEE Transactions on Industrial Electronics, 63(3), 1793-1803, (2016). https://dx.doi.org/10.1109/TIE.2015.2509913
8 Wang, Dong. Some further thoughts about spectral kurtosis, spectral, L2/L1, norm, spectral smoothness index and spectral Gini index for characterizing repetitive transients, Mechanical Systems and Signal Processing, 108, 360-368, (2018). https://dx.doi.org/10.1016/j.ymssp.2018.02.034

9 Papadaniil, C D., and Hadjileontiadis, L J. Efficient Heart Sound Segmentation and Extraction Using Ensemble Empirical Mode Decomposition and Kurtosis Features, IEEE Journal of Biomedical and Health Informatics, 18(4), 1138-1152, (2014). https://dx.doi.org/10.1109/JBHI.2013.2294399

10 Li, Y., Yang, Y., and Li, G, et al. A fault diagnosis scheme for planetary gearboxes using modified multi-scale symbolic dynamic entropy and mRMR feature selection, $M e-$ chanical systems and signal processing, 91(7), 295-312, (2017). https://dx.doi.org/10.1016/j.ymssp.2016.12.040

11 Wu, Y., Shang P., and Li, Y. Modified generalized multiscale sample entropy and surrogate data analysis for financial time series, Nonlinear Dynamics, 92(3), 1335-1350, (2018). https://dx.doi.org/10.1007/s11071-018-4129-x

12 Muhammad, Y., and Bong-Hwan K. Data Decomposition Techniques with Multi-Scale Permutation Entropy Calculations for Bearing Fault Diagnosis, Sensors, 18(4), 12781241, (2018). https://dx.doi.org/10.3390/s18041278

13 Tiwari, R., Gupta, V K., and Kankar, P. Bearing fault diagnosis based on multi-scale permutation entropy and adaptive neuro fuzzy classifier, Journal of Vibration and Control, 21(3), 461-467, (2015). https://dx.doi.org/10.1177/1077546313490778

14 Sun, D J., Wang, B, and Hu, X., et al. An Online Degradation Feature Extraction Technique for Shore Bridge Gearbox Based on Morphological Fractal Dimension and Sliding Window Weibull Fitting, Shock and Vibration, 20, 1-9, (2019). https://dx.doi.org/10.1155/2019/9216809

15 Bige, Y., and Tingting, Z. Multiscale base-scale entropy analysis of heart rate variability signal, Acta Physica Sinica, 60(7), 1729-1736, (2011). https://dx.doi.org/10.7498/aps.60.078701

16 Xin, L., Xiaoying, Q., and Xiaoqi, S., et al. An Improved Multi-Scale Entropy Algorithm in Emotion EEG Features Extraction, Journal of Medical Imaging and Health Informatics, 7(2), 436-439, (2017). https://dx.doi.org/10.1166/jmihi.2017.2031

17 Xu, F., Fang, Y J., Wu, Z., et al. A method based on multiscale base-scale entropy and random forests for roller bearings faults diagnosis, Journal of Vibroengineering, 20(1), (2018). https://dx.doi.org/10.21595/jve.2017.17153

18 Liangming, Z., Weicheng, C., Fault Diagnosis of Bearing based on Local Characteristic-scale Decomposition and Base-scale Entropy, Journal of Mechanical Transmission, 41(9), 183-188, (2017). https://dx.doi.org/10.16578/j.issn.1004.2539.2017.09.037

19 Vikhe, P S., Thool, V R., Morphological operation and scaled Reyni entropy based approach for masses detection in mammograms, Multimedia Tools and Applications, 77(18), 23777-23802, (2018). https://dx.doi.org/10.1007/s11042-018-5681-6

20 Mao, W., Feng, W., and Liang, X. A novel deep output kernel learning method for bearing fault structural diagnosis, Mechanical Systems \& Signal Processing, 117, 293-318, (2019). https://dx.doi.org/10.1016/j.ymssp.2018.07.034 